\title{
Wound Healing Management Bioinformatics Approach
}

\author{
Oksana N Shevtsova ${ }^{1 *}$ and Shevtsova $\mathrm{VK}^{2}$ \\ ${ }^{1}$ Senior Researcher, Institute for Safety Problems of NPP of the National Academy of Sciences of Ukraine,Ukraine \\ ${ }^{2}$ Researcher, National Medical University of 0 o Bogomolets, Ukraine
}

Submission: March 15, 2018; Published: June 25, 2018

*Corresponding author: Oksana N Shevtsova, Senior Researcher, Institute for Safety Problems of NPP of the National Academy of Sciences of Ukraine, Ukraine, Tel: 380-044-525-24-72; Email: okshevtsova@gmail.com

\begin{abstract}
The proposed review aim is an attempt to consider the wound healing process as a bioinformatics multivariate mathematical-biologicalmedical problem and attract attention to creation of unified approach to wound healing management, particularly for optimization of medical treatment. Wound healing process is closely associated with scarring problem which has economical, psychological and therapeutic consequences. Vast investigation in medicine, pharmacology and biology leads to creation of very complex mathematical models and to accumulation of huge medical data array, which require correct interpretation. Nanotechnology and nanomaterials for medical and pharmaceutical purposes give people a lot of possibilities to find new peculiarities and rules how to improve/accelerate/modify some biological processes.
\end{abstract}

The cellular and molecular mechanisms underpinning tissue repair and its failure to heal are still poorly understood, and current therapies are limited. Poor wound healing after trauma, surgery, acute illness, or chronic disease conditions affects millions of people worldwide each year and is the consequence of poorly regulated elements of the healthy tissue repair response, including inflammation, angiogenesis, matrix deposition, and cell recruitment. Failure of one or several of these cellular processes is generally linked to an underlying clinical condition, such as vascular disease, diabetes, or aging, which are all frequently associated with healing pathologies. The search for clinical strategies that might improve the body's natural repair mechanisms will need to be based on a thorough understanding of the basic biology of repair and regeneration.

Keywords: Wound healing; Bioinformatics approaches; Biochemical factors; Cellular factors; Network approach; Mucous membranes; Hypertrophic scar; Strictures; Surgical wounds; Matrix synthesis; Angiogenesis; Fibronectin; Remodeling phase; Oxaloacetic acids; Alkaline phosphatise; Consuming proteins; Collagen fibers; Re-epithelization; Cancer angiogenesis; Protein interaction; Algorithm

Abbreviations: PDGF: Platelet-Derived Growth Factor; TGF- $\beta$ : Transforming Growth Factor- $\beta$; bFGF: Fibroblast Growth Factor; EGF: Epidermal Growth Factor; IGF: Insulin-Like Growth Factor; GAG: Glycosaminoglycans; ECM: Extracellular Matrix; WHA: Wound Healing Assay; RQSA: Robust Quantitative Scratch Assay; EMBL-EBI: European Molecular Biology Laboratory-European Bioinformatics Institute; KEGG: Kyoto Encyclopaedia of Genes And Genomes; ARACNE: Algorithm For The Reconstruction Of Accurate Cellular Networks

\section{Introduction}

By definition, a wound is a lesion with a breach of the integrity of the skin or mucous membranes in their entire thickness, with access for contamination of deeper tissues by micro-organisms contained in the air or on objects touching the wound surface. The problem of wound healing and wound healing has a centuries-old history [1-3]. The highly orchestrated sequence of events that occur during normal, acute wound healing have been known for a very long time [1]. Likewise, the pathologic features that are characteristic of delayed wound healing as observed in chronic pressure ulcers, diabetic wounds, and venous stasis ulcers are also well known. On the opposite end of the spectrum the aberrant over healing seen in fibrotic responses such as keloid, hypertrophic scar, strictures, and adhesions are well described on a pathologic level. The various cells, cytokines, and the spectrum of enzymes that participate in both normal and pathologic wound repair are also well known.
However, the specific signalling switches that control all of these various normal and abnormal responses are unknown [1].

Taking into account the type, size and nature of skin wounds, wounds of internal and hollow internal organs, the effect of age on scar formation [4-6], the effect of primary treatment and therapies used, sutures and dressings and methods of treatment, the influence of co-morbidities (diabetes and etc.) and other factors [7]. It is obvious that the problem of wound healing is a complex and multi-parametric problem. It is understandable that the number of studies on wound healing, taking into account historical works and the number of monthly publications in modern periodicals is enormous. For example, in the US National Centre for Biotechnology, Medical and Genetic Information [6], as of March 2017, there are 8,451 articles on diabetic wounds (12060 for diabetic foot ulcers) and 137,124 articles on diabetic foot ulcers wound healing as a whole ("wound healing") - and 
their number is constantly increasing. As a result, the articles become more and more highly specialized.

\section{Classification of wounds}

The wounds were long ago and fairly well classified in different countries according to various external features: size, depth, nature, contamination, localization, etc. In addition to the classification of the qualitative characteristics of the wound (by the nature of the damage, the mechanism of application, the depth of penetration of the wound channel, etc.), there are a number of other classifications [8]. For example, in the US, the classification is used by the degree of contamination (American College of Surgeons / Centres for Disease Control and Prevention (1985)) - from class 1 (clean) to class 4 - (contaminated, infected) [9], the International Red Cross's classification by size, depth, the presence of foreign inclusions, etc., (and each of the parameters $((\mathrm{E}, \mathrm{X}, \mathrm{C}, \mathrm{F}, \mathrm{V}, \mathrm{M})$ is assigned a certain numerical value) [10], and many others.

Classification of surgical wounds is an important prognostic risk factor for postoperative complications. Classification of wounds is also used for clinical economic analysis and the use of standard clinical protocols [11,12]. Due to the insufficient knowledge of the wound healing process, there is currently no single generally recognized wound classification and, according to surgeons, there can not be a single approach to their treatment. Nevertheless, due to the creation of a number of new research methods, well-known processes at the clinical macro level, such as wound inflammation, reparative regeneration, scarring and other healing stages, are now being studied in detail at the micro level. Currently, clinicians usually denominate 3 or 4 major phases of wound healing [13]. In any case, sharp (fresh) wounds heal in a certain order, including the stages of coagulation, inflammation, matrix synthesis, angiogenesis, fibroplasia, epithelization, contraction and remodeling of the scar [14-19].

It is important to understand that all these microscopic events are complex and are initiated and supported by biochemical mediators known as cytokines and growth factors. Therefore, successful treatment requires knowledge of the factors leading to disruption of wound healing. Wounds heal within a short (fixed) period of time, if the factors inhibiting wound healing are eliminated. Understanding the development of the wound healing process and those processes that occur at the level of the cellular and vascular response, as well as which acting agents macrophages, growth factors, collagen, etc. - are determinative at each stage of the process - it is possible to formulate the goal of clinical impact (targeting) at this particular stage of the wound healing process - suppression of infection, stimulation of angiogenesis, inhibition of collagenesis, and in accordance with the ongoing process, i.e. wound healing phase [20]. On the basis of available data one can make an informed decision on the choice of the active substance (antibiotics, antiseptics, stimulants of reparative processes, etc.), pharmaceutical dosage form (solution, gel, ointment, etc.), coverage (bandages, microgel coatings, nano- bandages, radiation-modified gels, etc.), as well as modern physiotherapy methods (hyperbaric oxygenation, ultrasound cavitation and others) [21-24].

\section{Main events of the wound healing phases}

The wound-healing process consists of four highly integrated and overlapping phases: hemostasis, inflammation, proliferation, and tissue [25]. These phases and their biophysiological functions occur in the proper sequence, at a specific time, and continue for a specific duration at an optimal intensity $[7,21]$. Main events of normal wound healing process include:

I. Vascular constriction, platelet aggregation, degranulation, and fibrin formation (thrombus) during the hemostasis phase;

II. Neutrophil infiltration, monocyte infiltration and differentiation to macrophage, lymphocyte infiltration during inflammation phase;

III. Re-epithelization, angiogenesis, collagen synthesis, extracellular matrix formation during proliferation phase;

IV. Collagen remodeling, vascular maturation and regression during remodeling phase.

V. There are many factors that can affect wound healing which interfere with one or more phases in this process, thus causing improper or impaired tissue repair.

\section{Wound healing scenario and main key players}

In adult humans, normal wound healing involves the following the stages [7]:

i. Rapid hemostasis;

ii. Appropriate inflammation;

iii. Mesenchymal cell differentiation, proliferation, and migration to the wound site;

iv. Suitable angiogenesis;

v. Prompt re-epithelialization (re-growth of epithelial tissue over the wound surface); and

vi. Proper synthesis, cross-linking, and alignment of collagen to provide strength to the healing tissue [21,25].

The phase of hemostasis begins immediately after wounding, with vascular constriction and fibrin clot formation. A blood clot, consisting of fibrin, fibronectin, vitronectin, von Willebrand factor and thrombospondin, provides a preliminary matrix for cell migration. In order to limit blood loss, there is a rapid vasoconstriction and activation of the blood coagulation cascade, which leads to the formation of clot and aggregation of platelets. Platelets in the clot are necessary for hemostasis, as well as for a normal inflammatory response. The clot and surrounding wound tissue release pro-inflammatory cytokines and growth factors. 
During the degranulation of platelets, vasoactive substances and growth factors are released, including platelet-derived growth factor (PDGF), transforming growth factor- $\beta$ (TGF- $\beta$ ), a major fibroblast growth factor (bFGF), epidermal growth factor (EGF), insulin-like growth factor (IGF), vascular endothelial growth factor VEGF, keratinocyte growth factor KGF, connective tissue growth factor, CTGF, etc. [17]. Once bleeding is controlled, inflammatory cells migrate into the wound (chemotaxis) and promote the inflammatory phase, which is characterized by the sequential infiltration of neutrophils, macrophages, and lymphocytes [25-27].

An important function of neutrophils is the clearance of invading microbes and cellular debris in the wound area, although these cells also produce substances such as proteases and reactive oxygen species, which cause some additional bystander damage. Macrophages play multiple roles in wound healing $[28,18]$. In the early wound, macrophages release cytokines that promote the inflammatory response by recruiting and activating additional leukocytes [29]. Macrophages are also responsible for inducing and clearing apoptotic cells (including neutrophils), thus paving the way for the resolution of inflammation. As macrophages clear these apoptotic cells, they undergo a phenotypic transition to a reparative state that stimulates keratinocytes, fibroblasts, and angiogenesis to promote tissue regeneration [30]. In this way, macrophages promote the transition to the proliferative phase of healing. T-lymphocytes migrate into wounds following the inflammatory cells and macrophages, and peak during the lateproliferative/early-remodeling phase. The role of T-lymphocytes is not completely understood and is a current area of intensive investigation. The proliferative phase generally follows and overlaps with the inflammatory phase, and is characterized by epithelial proliferation and migration over the provisional matrix within the wound (re-epithelialization).

Fibroblasts appear in the wound for 2-3 days after its formation and dominate among cell populations in the first week. The early extracellular matrix mainly consists of fibronectin and hyaluronates, serving as a wound bed, to which fibroblasts can migrate and fix. The sources of these fibroblasts are derivative of resting fibrocytes of the regional connective tissue and perivascular adventitia. Fibroblasts produce a variety of substances necessary for wound healing, including glycosaminoglycans (GAG) and collagen. Proteoglycans are proteins to which polysaccharides are attached. The four main glycosaminoglycans include hyaluronic acid, chondroitin4-sulfate, dermatin sulfate and heparin sulfate. They form an amorphous gel called "basic substance", which plays an important role in the deposition and aggregation of collagen fibrils [31,32]. In the reparative dermis, fibroblasts and endothelial cells are the most prominent cell types present and support capillary growth, collagen formation, and the formation of granulation tissue at the site of injury. Within the wound bed, fibroblasts produce collagen as well as glycosaminoglycans and proteoglycans, which are major components of the extracellular matrix (ECM). Following robust proliferation and ECM synthesis, wound healing enters the final remodeling phase, which can last for years.

In this phase, regression of many of the newly formed capillaries occurs, so that vascular density of the wound returns to normal. One critical feature of the remodeling phase is ECM remodeling to an architecture that approaches that of the normal tissue. The wound also undergoes physical contraction throughout the entire wound healing process, which is believed to be mediated by contractile fibroblasts (myofibroblasts) that appear in the wound $[25,26,19]$. Several different cell types are involved in the wound healing process, and, the cellular activities of any particular cell type may also vary during different stages of repair. The complexity and coordination of the healing process are major barriers to therapeutic approaches, since any therapeutic treatment must effectively be sequenced to the appropriate stage.

\section{Biochemical factors of healing process}

Woessner J \& Boucek R $[33,34]$ studying the dynamics of various enzymes in the development of granulation tissue, identified 26 of the most important enzymes for the process, which, according to their functional significance and phase of action, can be divided into three groups:

a) The first group of wound enzymes includes: transaminases of glutamic and oxaloacetic acids, imidopeptidase, catalase, cnthochrom-C-reductase and phenosulfatase. These enzymes participate in the processes of the initial phase of connective tissue regeneration and their quantity and activity grow if the DNA amount increases in damaged tissues.

b) The dynamics of activity of the second group of enzymes (peroxidase, lactide dehydrogenase, malicodehydraz) allowed the authors of the classification to determine their participation in the proliferation of cells of the emerging granulation tissue. Alkaline phosphatase also takes an active part in this process.

c) The third group of wound enzymes (proteinase, glucuronidase, acid phosphatase) is characterized by a maximum of activity in the relatively late stages of wound healing. For each enzyme, it is typical to achieve a maximum of activity after a certain period of time and maintain intensity for several days after injury.

Lysosomes participating in the wound process contain more than 30 enzymes that are actively involved even in the first hours after injury in the complex biochemical processes of wound catabolic and anabolic reactions. According to the studies of A A Pokrovsky \& V A Tutelyan [20], lysosomal enzymes play an important role in the adaptation reactions of the organism, aimed at adapting metabolic processes to a more adequate expenditure of intracellular protein reserves for reconstructive cell purposes, 
which provides an optimal expediency of consuming proteins. In addition, lysosomal enzymes provide the necessary level of phagocytic activity of leukocytes, that is, protective functions of the body.

Aminopeptidases in the wound take part in dissolving the damaged and altered collagen and cleaning the wound of destructed fragments of collagen fibers [32], while maintaining a sufficient aminoacid pool for the synthesis of newly formed collagen proteins. Transferases in the wound process participate in the phagocytic reactions of local and migrating cells, in the regulatory mechanisms of vasoconstrictive amines, keratinization of the healing, regenerating epithelium [31]. Glycosyltransferases are closely related to the metabolism of glycogen, which is the main carbohydrate reservoir of energy required for various biochemical reactions of the regeneration process and wound healing. An increase in the activity of oxidoreductases occurs in the early period of the wound process.

When the wound is healed, there is observed the regularity in the rise in the activity of nonspecific esterase. The first rise is associated with the multiplication and growth of fibroblasts. The second and third rises in the activity of this enzyme coincide in time and are therefore associated with the processes of formation of collagen fibers and converting them into coarse fibrous connective tissue. Each phase of the wound process (wound inflammation, regeneration, remodelling) is characterized by an increase in the activity of individual groups of enzymes specific for each wound healing stage. For example, the wound inflammation phase is started as a vascular reaction, during of which vasoactive substances are released, including proteolytic enzymes. The level of vasoconstrictive amines acting like local anti-inflammatory hormones is regulated by transferase. At the same time, an increase in monoamine oxidase activity in the first hours after injury hinders the excessive development of an inflammatory reaction, blocking the excessive activity of amines. Approximately after 6-9 hours after tissue damage, necrosis of the most injured tissues and cells is started in the central zone of the wound. The early indication of necrosis is the decrease or disappearance of enzyme activity.

Enhancement of enzymatic activity in the wound occurs not only due to activation of enzyme systems, but also due to an increase in the concentration of enzymes originating from blood plasma, migrating leukocytes and from local cellular elements. During the first 12-16 hours, a large number of neutrophilic polymorphonuclear leukocytes appear in the wound, in the decay of which a large number of enzymes are released from the granules into the extracellular space, which contribute to the destruction and removal of tissue decomposition components from the wound. At the same time, high activity of glucose-6phosphate dehydrogenase is observed in mononuclear cells, which corresponds to the initial stage of the pentose phosphate cycle, associated with phagocytosis and antibacterial action of oxidative reactions. The remodeling phase is started with the migration of epithelial cells. The energy required for this process depends on the enzymatic activity of ATPase.

Fibroblasts are responsible for the synthesis of polysaccharides and collagen in the regeneration phase. These cells, along with leukocytes, extracellular substance and newly formed vessels, determine the level of enzymatic activity of the granulation tissue. In the remodeling phase (approximately on the 12th-14th day of the wound process, the activity of most of the enzymes is decreased, and then completely stopped. Thus, metabolism of the wound healing process is described by various types of catabolic and anabolic enzymatic reactions. Wound healing depends on the timely activation of the necessary enzyme systems, the intensity and duration of their action. The biochemistry of wound healing consists of multiple pathways and cascades that traverse through the several stages of wound healing in an orderly progression leading toward tissue replacement and a healed wound. Hemostasis and the acute inflammatory response set into motion biochemical signaling that leads to re-epithelization and wound granulation tissue formation. Cytokines, chemoattractants, and growth factors recruit and activate cells. Proteases are activated and released to promote cell migration and connective tissue remodeling. Extracellular matrix molecules are deposited first to form a scaffold supporting the cells and blood vessels of granulation tissue, followed by deposition and remodeling of collagenous connective tissue. Resolution, or down regulation, of healing occurs as levels of activating signals decrease and de-activating signals increase [35].

In a chronic wound, biochemical signalling pathways may be disrupted in one of a number of ways. Underlying pathologies may produce a wound environment with inappropriate expression of signalling molecules or responding cells, thus preventing the normal progression of healing. Bacterial burden may overwhelm the wound environment, shifting the $\mathrm{pH}$ and releasing metabolic inhibitors or toxins as well as inducing chronic inflammation detrimental to healing. Chronic wounds may eventually exhaust their supply of activating signals and become stalled in the healing process [36]. The main moments of wound healing are simultaneously ongoing interrelated processes of filling the wound defect with granulations, gradual covering of the wound surface with regenerating epithelium and concentric wound tightening during maturation of granulations and their transformation into scar tissue. The essence of contraction consists in shifting the edges of the wound to the centre of the wound defect. The role of contraction in closing the wound defect depends on the degree of mobility of the surrounding wound of the skin. Healing of wounds located on sites with a sluggish skin, proceeds mainly due to tissue growth and epithelialization from the edges of the wound defect, whereas in the healing of the wounds of the mobile skin, the process of contraction is of great importance. Therefore, studying the healing rate of a planar wound, it is advisable to study separately the rate of epithelialization and contraction. The speed of wound healing is 
not just a function of time, but it reflects the stage of the wound process, the completeness and harmony of the realization of its mechanisms. Therefore, mathematical modeling of the dynamics of wound healing is a very effective method of studying the wound process and can play an important role in studies to optimize the treatment of wounds.

\section{Wound healing bioinformatics tools}

There are many bioinformatics tools. We review two the most interesting bioinformatics tools from the wound healing point of view $[23,24]$.

\section{Laverne bioinformatics tool}

Laverne is a handy bioinformatics tool to help facilitate scientific exploration of related genes, diseases and pathways based on co-citations [23]. Disease and disorder research has been conducted in relation to the Wound Healing Pathway and Inflammation, Neoplasms, Tumor Angiogenesis, Malignant Neoplasms, Ulcer. The study of the Wound Healing Pathway has been mentioned in research publications which can be found using Laverne bioinformatics tool. The Wound Healing Pathway has been researched in relation to Angiogenesis, Regeneration, Cell Migration, Cell Proliferation, Secretion.

\section{Robust quantitative scratch assay}

Since cell migration is significant in repair processes and disease advancement such as metastasis and cancer angiogenesis, it can be used as a parameter to describe the state of health of various cell lines $[28,29]$. Furthermore, quantification of cell migration can be used to measure the effect of drug treatments on the same cell line. The Wound Healing Assay (WHA) is used to quantify motility by monitoring the evolution of a wounded monolayer of cells. The experimental set-up consists of growing a confluent monolayer of cells under specific conditions (i.e. treated or not treated with a drug), then creating a wound or scratch on each layer/well, and then imaging all wounds multiple times over a period of time. Since the rate how wounds close may depend on conditions used, measuring wound areas from individual images are used to estimate migration rates, and thus quantify condition-specific cell motility. In order to obtain reliable results, it is necessary to measure wound areas across multiple replicates of the same cell condition, and record multiple frames per replicate to determine whether changes are statistically significant. To make this task feasible, an automated system is required to process the high volumes of data and make measurements independent of image artefacts such as uneven illuminations, smudges or scratches on the well plate lid, in addition to some variation of initial wound width and shape [37].

The wound healing assay (or scratch assay) is a technique frequently used to quantify the dependence of cell motility - a central process in tissue repair and evolution of disease - subject to various treatments conditions. However processing the resulting data is a laborious task due its high throughput and variability across images. The Robust Quantitative Scratch Assay algorithm introduced statistical outputs where migration rates are estimated, cellular behaviour is distinguished and outliers are identified among groups of unique experimental conditions. The Robust Quantitative Scratch Assay (RQSA) algorithm has been developed using MATLAB 7.11.0 to address the challenges imposed by the large datasets, variability in image illuminations and implemented a statistical output for an improved quantification of cell motility using the wound healing assay. The algorithm requires MATLAB 7.11.0 and uses the Statistics and Image Processing Toolboxes. The estimated migration rates for all wells in a single condition are grouped, then outliers are flagged and discarded before calculating the average migration rate for each experimental condition [36].

\section{A network approach to wound healing}

A network approach to wound healing looks as a challenging approach, joining various cells, cytokines, and the spectrum of enzymes that participate in both normal and pathologic wound repair at the assumption that this huge data array is well known. What is the question: how to define the specific signaling switches that control all of these various normal and abnormal responses? And how we can use available knowledge to improve wound healing. Network analysis is an innovative strategy that has the potential to be used to better characterize the molecular switches that control normal and abnormal wound healing mechanisms [38]. Authors of paper have reviewed some attempts to built a network approaches. There are two broad categories of methods with high level of detail - data-driven and knowledge-based methods. How to identify curing diseases and injuries applying network approach? The main distinction is in the role the network plays in the analysis. Data-driven methods focus on network inference, with the network being the result of analyzing the data. The knowledge-based approaches start with a large structure of connections reflecting current knowledge of biology, gathered from databases and literature. That background knowledge serves as a basis for deriving a focused network that is related to the results of the experiment under study [1]. Knowledge-based methods also use experimental data, but they interpret them in the context of information taken from the online accessible databases. The essence of knowledge-based methods is not to discover new links, but to find out which subset of known connections form a sub network that differentiates between phenotypes, disease states, or timepoints.

There are many online accessible databases, algorithms, methods which can be used for creation of relevance networks, for example PINA (protein interaction network analysis) database for protein-protein interactions [38]; Intact-the European Molecular Biology Laboratory-European Bioinformatics Institute (EMBL-EBI) Protein Interaction DB [39]; the BioGRID (biological general repository for interaction datasets) database for genetic and physical interactions [40]; KEGG (Kyoto encyclopedia of genes and genomes) for metabolic information [41]. Some of the 
best professional software packages for network analysis, such as Ingenuity and Pathway Studio, also provide their databases for analysis with their proprietary software [42,43]. Another popular software package for network analysis is Cytoscape, which offers a number of additional functions (plugins) and is publicly available [44].

The ARACNE (algorithm for the reconstruction of accurate cellular networks) method was proposed as an improvement to relevance networks $[45,46]$. As the final step of the analysis, the networks produced from experimental or literature data can be further treated to include the closest neighborhood of the most important nodes or to find common regulators and common targets of the genes/proteins/microRNAs which these nodes represent. In such a way, predictions can be made for other genes involved in the disease of interest and its curing, and novel drug candidates can be identified. All this will bring medicine much closer to individualized and more efficient treatments. A comparative analysis of some of these tools is presented in [47].

\section{Experimental data for network analysis}

The problem that affects network methods is the small sample size of typical experiments. This is a problem for analysis of profiling experiments in general, since the number of observed variables vastly exceeds the number of samples, but it becomes even more pronounced in methods that aim at inferring networks from data. Network analysis relies on experimental measurements of quantities that build the network. Most often, these are captured using profiling experiments [37]. Soulet et al. [48] relied on transcriptomic profiling using Affymetrix GeneChip microarray in their exploration of healing of wounds in the chick embryo model. Transcriptomic profiling has the advantage of capturing genome-wide profile of gene expression in a single experiment, but the results often require confirmation using quantitative real-time polymerase chain reaction (qPCR) for selected small subset of identified genes. Other studies of wound samples also rely on the combination of transcriptomic profiling using microarrays with qPCR validation step. Measurements at the protein level show a more direct view of molecular processes involved in wound healing [49-51]. The methods used in analyzing results from profiling studies oriented on wound healing are currently limited mostly to enrichment analysis using gene ontology terms, and to clustering. However, papers that take a more detailed look at networks involved in response to wounds are emerging. Authors of [37] have reviewed two such papers $[52,48]$, one focused on proteomics and another on transcriptomic profiling.

A multi-modal proteomic profiling study by Edsberg et al. [52] focused on pressure ulcers, tracked over a time span of up to 6 weeks in 32 subjects, with wound fluid samples collected from the interior and separately from the periphery of the wounds. The network analysis was performed using Ingenuity, and focused on two aspects: uncovering differences in interior wound fluid between chronic and healed wounds, and, within chronic wounds, the differences between fluid samples collected from interior and periphery of the wound. Matrix metalloproteinases are more abundant in wounds that eventually healed, while tissue inhibitors of metalloproteinases and proinflammatory cytokines are more abundant in chronic wounds. Keratins and members of the S100 family are significantly more abundant in periphery of chronic wounds, while some matrix metalloproteinases and proinflammatory cytokines are overabundant in wound interior. One other molecular species that was pointed by network analysis is fibrinogen. The relationship of fibrinogen to differences between chronic and healing wounds has been observed in other studies, with beta and gamma chains significantly more abundant in ulcers $[53,54]$.

\section{Conclusion}

The network approach is a paradigm that has been successfully applied to the study of regulation and signaling. The articles reviewed here are among the first efforts to bring networks into the study of wound healing. The innovation from applying network methods comes from its ability to enhance understanding of high-throughput proteomic or transcriptomic profiling. Ultimately, network approaches can help for a deeper, integrated understanding of the wound healing process and make it more optimal. As this review shows, the application of network methods to basic research in wound healing is only in its beginning. Future profiling studies will benefit from incorporating systems biology approaches to data analysis. The complexity of such multi-modal datasets makes the network approach an important tool. The network approach to wound healing is still in the developmental stage. Once the process is better understood, it is hoped to become a valuable tool for clinicians, enabling the treatment of many pathologic wound healing responses, such as fibrosis and chronic, nonhealing wounds.

\section{References}

1. Diegelmann RF, Evans MC (2004) Wound healing: an overview of acute, fibrotic and delayed healing. Front Biosci 9: 283-289.

2. Mironov VI, Frolov AP, Gileva II (2010) Theory of wounds. History, development, perspectives. Part I, Sibirskiy-Meditsinskyi-zhurnalIrkutsk,4(95): 118-122.

3. Mironov VI, Frolov AP, Gileva II (2010) Theory of wounds. History, development, perspectives. Part II, Sibirskiy-Meditsinskyi-zhurnalIrkutsk 96: 134-138.

4. Ferguson MWJ, O'Kane S (2004) Scar-free healing: from embryonic mechanisms to adult therapeutic intervention. Philos Trans R Soc Lond B Biol Sci 359(1445): 839-850.

5. Degen KE, Gourdie RG (2012) Embryonic Wound Healing: A Primer for Engineering Novel Therapies for Tissue Repair. Birth Defects Res C Embryo Today 96(3): 258-270.

6. Ehrlich HP, Desmoulière A, Diegelmann RF, Cohen IK, Compton CC, et al. (1994) Morphological and immunochemical differences between keloid and hypertrophic scar. Am J Pathol 145(1):105-113. 


\section{Biostatistics and Biometrics Open Access Journal}

7. Guo S, DiPietro LA (2010) Factors Affecting Wound Healing. J Dent Res 89(3): 219-229.

8. The National Center for Biotechnology Information advances science and health by providing access to biomedical and genomic information.

9. Mangram AJ, Horan TC, Pearson ML, Silver LC, Jarvis WR (1999) The Hospital Infection Control Practices Advisory Committee. Guideline for prevention of surgical site infection, 1999. Am J Infect Control 20(4): 250-278.

10. https://en.wikipedia.org/wiki/International_Red_Cross_Wound Classification_System

11. Devaney L, Rowell KS (2004) Improving Surgical Wound ClassificationWhy it Matters. AORN J 80(2): 208-289.

12. Zinn J, Swofford V (2014) Quality-improvement initiative: Classifying and documenting surgical wounds Interprofessional collaboration promoted a successful initiative to improve wound classification. Wound Care Advisor 3(1): 32-38.

13. Kerstein MD (1997) The scientific basis of healing. Adv Wound Care 10(3): 30-36.

14.http://www.worldwidewounds.com/2004/august/Enoch/ Pathophysiology-of-Healing.htm

15. www.mhhe.com/prentice15e

16. Harding KG, Morris HL, Patel GK (2002) Science, medicine, and the future. Healing chronic wounds BMJ 324: 160-163.

17. Enoch S, Price PE (2004) Cellular, molecular and biochemical differences in the pathophysiology of healing between acute wounds, chronic wounds and wounds in the aged. World Wide Wounds.

18. Leibovich SJ, Ross R (1975) The role of the macrophage in wound repair. A study with hydrocortisone and antimacrophage serum. Am J Pathol 78(1): 71-100.

19. Grinnell F(1994) Fibroblasts, myofibroblasts and wound contraction. Cell Biol 124(4): 401-404.

20. Pokrovsky AA, Zbarsky IB, Tutelyan VA, Perevoshchikova KA, Lashneva NV, et al. (1968) Dokl Akad Nauk SSSR (Biochemistry) 181:1280.

21. Mathieu D, Linke JC, Wattel F (2006) Non-healing wounds. In: Mathieu DE (Eds), Handbook on hyperbaric medicine, Springer, Netherlands, 401-427.

22. Keast D, Orsted H (2011) Basic Principles of Wound Healing. Wound Care Canada 9(2): 4-12

23. https://www.novusbio.com/pathways/wound-healing

24. Andrea V, Angeli C, Pastrello R, McQuaid H, Li Andrea J, et al. (2016) Robust quantitative scratch assay. Bioinformatics 32(9): 1439-1440.

25. Gosain A, DiPietro LA (2004) Aging and wound healing. World J Surg 28(3): 321-326

26. Campos AC, Groth AK, Branco AB (2008) Assessment and nutritional aspects of wound healing. Curr Opin Clin Nutr Metab Care 11(3): 281288

27. Broughton G, Janis JE, Attinger CE (2006) The basic science of wound healing. Plast Reconstr Surg 117(7 Suppl): 12S-34S.

28. Gebäck T, Schulz MM, Koumoutsakos P, Detmar M (2009) TScratch: a novel and simple software tool for automated analysis of monolayer wound healing assays. Biotechniques 46(4): 265-274.

29. Zordan MD, Mill CP, Riese DJ, Leary JF (2011) A high throughput, interactive imaging, bright-field wound healing assay. Cytometry A 79(3): 227-232
30. Edwards JP, Zhang X, Frauwirth KA, Mosser DM (2006) Biochemical and functional characterization of three activated macrophage populations. J Leukoc Biol 80(6): 1298-1307.

31. Raekallio J (1960) Enzymes Histochemically Demonstrable in the Earliest Phase of Wound Healing. Nature 188: 234-235.

32. Ross R, Odland G (1968) Human wound repair. II. Inflammatory cells, epithelial-mesenchymal interrelations, and fibrogenesis. J Cell Biol 39(1): 152-168.

33. Woessner JF, Boucek RJ (1961) The Determination of Hydroxyproline in tissue and protein samples containing small propotions of this Amino acids. Arch Biochem Biophys 93: 85.

34. Woessner JF, Boucek RJ (1961) Arch Biochem Biophys 93: 95.

35. Patricia A (2003) Biochemistry of Wound Healing. Wound 15:11.

36. Ge K, Wu JJ, Qian L, Wu MJ, Wang FL, et al. (2015) Bioinformatic analysis of the effect of type II diabetes on skin wound healin. Genet Mol Res 14(2): 4802-4811.

37. Arodz T, Bonchev D, Diegelmann RF (2013) A Network Approach to Wound Healing. Adv Wound Care (New Rochelle) 2(9): 499-509.

38. Wu J, Vallenius T, Ovaska K, Westermarck J, Mäkelä TP, et al. (2000) Integrated network analysis platform for protein-protein interactions. Nat Methods 6(1): 75-77.

39. Kerrien S, Aranda B, Breuza L, Bridge A, Broackes-Carter F, et al. (2011) The IntAct molecular interaction database in 2012. Nucleic Acids Res 40: D841-840.

40. Stark C, Breitkreutz BJ, Chatr-Aryamontri A, Boucher L, Oughtred R, et al. (2011) The BioGRID interaction database: 2011 update. Nucleic Acids Res 39: D698-704.

41. Kanehisa M, Goto S, Sato Y, Furumichi M, Tanabe M (2012) KEGG for integration and interpretation of large-scale molecular datasets. Nucleic Acids Res 40:D109-104.

42. www.ingenuity.com

43. Nikitin A, Egorov S, Daraselia N, Mazo I (2003) Pathway Studiothe analysis and navigation of molecular networks. Bioinformatics. 19(16): 2155-2157

44. Shannon P, Markiel A, Ozier O, Baliga NS, Wang JT, et al. (2003) Cytoscape: a software environment for integrated models of biomolecular interaction networks. Genome Res 13(11): 2498-2504.

45. Margolin AA, Nemenman I, Basso K, Wiggins C, Stolovitzky G, et al. (2006) ARACNE: an algorithm for the reconstruction of gene regulatory networks in a mammalian cellular context. BMC Bioinformatics 7(Suppl 1): S7.

46. Margolin AA, Wang K, Lim WK, Kustagi M, Nemenman I, et al. (2006) Reverse engineering cellular networks. Nat Protoc 1(2): 662-671.

47. Thomas S, Bonchev D (2010) A survey of current software for network analysis in molecular biology. Hum Genomics 4(5): 353-360.

48. Soulet F, Kilarski WW, Antczak P, Herbert J, Bicknell R, et al. (2010) Gene signatures in wound tissue as evidenced by molecular profiling in the chick embryo model. BMC Genomics 11: 495.

49. Chen L, Arbieva ZH, Guo S, Marucha PT, Mustoe TA, et al. (2010) Positional differences in the wound transcriptome of skin and oral mucosa. BMC Genomics 11: 471.

50. Hardman MJ (2008) Ashcroft GS. Estrogen, not intrinsic aging, is the major regulator of delayed human wound healing in the elderly. Genome Biol 9(5): R80. 
51. Roy S, Khanna S, Rink C, Biswas S, Sen CK (2008) Characterization of the acute temporal changes in excisional murine cutaneous wound inflammation by screening of the wound-edge transcriptome. Physiol Genomics. 34(2):162-184.

52. Edsberg LE, Wyffels JT, Brogan MS, Fries KM (2012) Analysis of the proteomic profile of chronic pressure ulcers. Wound Repair Regen 20(3): 378-401.

This work is licensed under Creative Commons Attribution 4.0 Licens

DOI: 10.19080/BBOJ.2018.07.555709
53. Roy S, Sen CK (2012) miRNA in wound inflammation and angiogenesis. Microcirculation 19(3):224-232.

54. Eming SA, Koch M, Krieger A, Brachvogel B, Kreft S, et al. (2010) Differential proteomic analysis distinguishes tissue repair biomarker signatures in wound exudates obtained from normal healing and chronic wounds. J Proteome Res 9(9): 4758-4766.

\section{Your next submission with Juniper Publishers will reach you the below assets}

- Quality Editorial service

- Swift Peer Review

- Reprints availability

- E-prints Service

- Manuscript Podcast for convenient understanding

- Global attainment for your research

- Manuscript accessibility in different formats

( Pdf, E-pub, Full Text, Audio)

- Unceasing customer service

Track the below URL for one-step submission https://juniperpublishers.com/online-submission.php 